Article

\title{
Cerebral White Matter Lesions on Diffusion-Weighted Images and Delayed Neurological Sequelae after Carbon Monoxide Poisoning: A Prospective Observational Study
}

\author{
Sangun Nah ${ }^{1}{ }^{1}$, Sungwoo Choi ${ }^{1}$, Han Bit Kim ${ }^{1}$, Jungbin Lee ${ }^{2}$, Sun-Uk Lee ${ }^{3} \mathbb{1}$, \\ Young Hwan Lee ${ }^{1}$, Gi Woon Kim ${ }^{1}$ and Sangsoo Han ${ }^{1, *(1)}$ \\ 1 Department of Emergency Medicine, Soonchunhyang University Bucheon Hospital, Bucheon 14584, Korea; \\ potter325@naver.com (S.N.); csw3613@naver.com (S.C.); hanbit6105@gmail.com (H.B.K.); \\ emer0716@naver.com (Y.H.L.); emer0325@naver.com (G.W.K.) \\ 2 Department of Radiology, Soonchunhyang University Bucheon Hospital, Bucheon 14584, Korea; \\ noiese@schmc.ac.kr \\ 3 Department of Neurology, Korea University Medical Center, Seoul 02841, Korea; furyrage@hanmail.net \\ * Correspondence: brayden0819@daum.net; Tel.: +82-32-621-5116
}

Received: 29 August 2020; Accepted: 14 September 2020; Published: 16 September 2020

check for updates

\begin{abstract}
Introduction: Carbon monoxide (CO) poisoning can result in delayed neurological sequelae (DNS). Factors predicting DNS are still controversial. This study aims to determine whether acute brain lesions observed using diffusion-weighted magnetic resonance imaging (MRI) following acute $\mathrm{CO}$ poisoning are related to the subsequent development of DNS. Methods: This prospective study was conducted on patients with $\mathrm{CO}$ poisoning treated at a university hospital in Bucheon, Korea. From August 2016 to July 2019, a total of 283 patients visited the hospital because of CO poisoning. Exclusion criteria included age under 18 years, refusing hyperbaric oxygen therapy, refusing MRI, being discharged against medical advice, being lost to follow-up, having persistent neurological symptoms at discharge, and being transferred from another hospital $24 \mathrm{~h}$ after exposure. Results: Of the 154 patients included in the final study, acute brain lesions on MRI (ABLM) were observed in 49 patients (31.8\%) and DNS occurred in 30 patients (19.5\%). In a logistic regression analysis, lower Glasgow coma scale score and higher exposure time were associated with DNS, and the presence of ABLM in white matter was significantly associated with DNS (OR 6.741; 95\% CI, 1.843-24.660; $p=0.004)$. Conclusion: The presence of ABLM in white matter was significantly related to the occurrence of DNS. Early prediction of the risk of developing DNS through MRI may be helpful in treating patients with $\mathrm{CO}$ poisoning.
\end{abstract}

Keywords: carbon monoxide poisoning; magnetic resonance imaging; neurotoxicity; white matter

\section{Introduction}

Carbon monoxide ( $\mathrm{CO}$ ) is a colorless, tasteless, and odorless toxic gas produced by incomplete combustion of carbon-based fuel and material [1]. Because of these characteristics, patients often do not know they are affected and, in mild cases, diagnosis and treatment are often delayed because symptoms do not appear immediately. CO has 250 times greater affinity for hemoglobin $(\mathrm{Hb})$ than oxygen, so it binds $\mathrm{Hb}$ and reduces oxygen transport. This is a major cause of mortality and morbidity associated with $\mathrm{CO}$ poisoning because this process can damage the brain and heart, which are vulnerable to ischemia [2]. 
DNS is one of the common sequelae of brain damage caused by CO poisoning, which can occur two days to six weeks after successful treatment for acute $\mathrm{CO}$ poisoning. DNS should be suspected if symptoms such as memory loss, movement disorder, and/or dementia appear [3]. Previous studies have suggested that old age, loss of consciousness at the time of exposure, and initial neurological abnormality are predictors of DNS following acute CO poisoning, but this remains controversial $[4,5]$. The predictive values of these factors appear inconsistent because of differences in types of patients, severity of $\mathrm{CO}$ poisoning, and diversity of measurement results [6].

Magnetic resonance imaging (MRI) is one of the most accurate tests for diagnosing ischemic brain lesions and brain diseases, such as demyelinating diseases, Alzheimer's disease, and epilepsy [7,8]. In addition, one of the best ways to present acute ischemic brain lesions is MRI diffusion-weighted images (DWIs) [9,10]. The pathophysiology of CO poisoning results in apoptosis, abnormal inflammatory responses, and ischemia-reperfusion injury in the brain, which can cause ischemic brain tissue damage and demyelination of white matter [3]. Therefore, early ischemic lesions in $\mathrm{CO}$ poisoning can be found using MRI, especially DWIs. Previous studies reported that white matter hyperintensities and hippocampal atrophy were usually found on brain MRI in CO poisoning [11,12]. In other studies, the putamen, thalamus, and caudate nucleus were found to be affected, appearing as asymmetric focal hyperintense lesions in T2-weighted and FLAIR images [13,14]. Globus pallidus is also among the regions that are vulnerable to $\mathrm{CO}[12,15]$. Like this, various brain lesions can be found in $\mathrm{CO}$ poisoning but there is no consensus on the MRI findings that are related with the development of DNS yet.

This study investigated the association of acute brain lesion on MRI (ABLM), particularly on DWIs, with the occurrence of DNS. The relationship between patterns or locations of ABLMs and the occurrence of DNS was also investigated.

\section{Methods}

\subsection{Study Design and Setting}

This prospective observational study was conducted using a CO registry and telephone interviews with all CO poisoning patients who visited the university hospital emergency department (ED) located in Bucheon, Korea. This study was approved by the hospital institutional trial review board (IRB file No. 2020-03-019).

\subsection{Participant Selection}

Since August 2016, all patients visiting the ED of our institution with CO poisoning have been placed on the $\mathrm{CO}$ registry. All patients who had adequate history or physical examination after $\mathrm{CO}$ poisoning, and measured carboxyhemoglobin $(\mathrm{COHb})$ values $>5 \%$ in non-smokers and $>10 \%$ in smokers upon the first visit to the ED were considered to be suffering from $\mathrm{CO}$ poisoning. The present study was conducted on CO poisoning patients who visited from August 2016 to July 2019. We included patients who consented to hyperbaric oxygen (HBO) therapy. Patients were excluded if they were under 18 years of age, refused HBO therapy, refused MRI, were discharged against medical advice, were lost to follow-up, had neurological deficits persisting at discharge from the ED, and/or were transferred from another hospital $24 \mathrm{~h}$ after exposure (Figure 1). According to our management protocol, patients were scheduled for MRI scans within 2 days of visiting the ED. However, MRI was not performed when patients or their proxy did not consent or when a medical condition contraindicated MRI scan. 


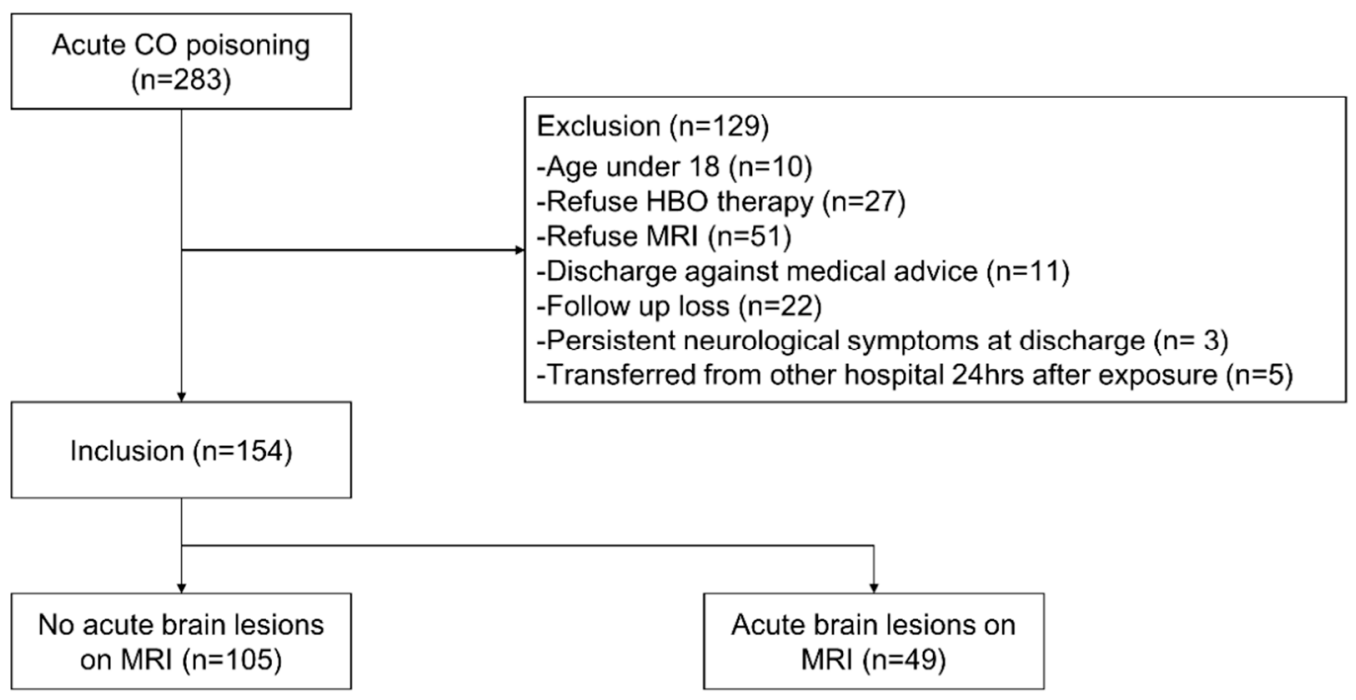

Figure 1. Flow chart of patient selection. Abbreviations: $\mathrm{CO}$, carbon monoxide; HBO, hyperbaric oxygen; MRI, magnetic resonance imaging.

\subsection{HBO Therapy Indication and Protocol}

$\mathrm{HBO}$ therapy was delivered if the patient's initial $\mathrm{COHb}$ was $\geq 25 \%$ (COHb $\geq 15 \%$ in pregnant women), there was a history of loss of consciousness or presence of neurological abnormalities regardless of $\mathrm{COHb}$ concentration, and/or there was apparent cardiac injury, such as abnormal electrocardiogram or elevation of troponin I. HBO therapy cannot be applied to endotracheal intubated patients in principle; however, when there were signs of spontaneous breathing and stabilization of vital signs, HBO therapy was delivered. HBO therapy was applied in a monochamber, and treatment was conducted in three sessions at intervals of $6-12 \mathrm{~h}$ within a day. In the first session, the total duration of HBO therapy was $150 \mathrm{~min} /$ session, and the target pressure was 3 atmospheres (absolute). For later sessions, they were aimed for $120 \mathrm{~min} /$ session and 2 atmospheres [16].

\subsection{Clinical and Laboratory Assessments}

At the ED visit, we collected the following data for our registry: demographic data, medical comorbidities, vital signs, Glasgow coma scale (GCS), CO exposure time, intentionality of poisoning, symptoms, and laboratory results. We also prospectively collected neurological symptoms and signs at discharge. DNS were defined as neurological abnormalities that occurred within 3 months of discharge and comprised symptoms such as insomnia, headache, dizziness, Parkinson-like syndrome, movement disorder, conscious disorder, mood disorder, and memory disorder [3,17]. Patients and their proxies were educated about DNS and discharged after receiving relevant documents. Patients visited the ED 1 month after discharge to see an emergency medicine specialist. In addition, telephone interviews about the presentation of DNS were performed with all patients at 2 weeks, and 3 months after discharge. If patients were suspected having DNS symptoms, they were re-admitted and finally diagnosed with DNS through performing additional brain MRI and consulting with neurologists.

\subsection{Imaging Analysis}

MRI examination was performed with a 3-T MRI unit (Signa HDXT 3.0; GE Healthcare, Chicago, IL, USA) using a standard head coil. The DWI parameters were as follows: repetition time, $9000 \mathrm{~ms}$; echo time, $77.5 \mathrm{~ms}$; matrix number, $192 \times 192$; field of view, $200 \mathrm{~mm} ; 2 \mathrm{~b}$ values of 0 and $1000 \mathrm{~s} / \mathrm{mm}^{2}$; slice thickness, $5 \mathrm{~mm}$; and interslice gap, $1 \mathrm{~mm}$. DWIs and automatically generated apparent diffusion coefficient map were studied $\left(b=1000 \mathrm{~s} / \mathrm{mm}^{2}\right)$. DWI was evaluated as follows: (1) presence of pathology, (2) number of pathologies, (3) asymmetry, and (4) location of pathology. Because the size, shape, and distribution of lesions varied, we categorized ABLMs into three patterns: globus 
pallidus lesions for globus pallidus; diffuse lesions for diffuse symmetric lesions; and focal lesions for asymmetric focal lesions. The presence of ABLM was also categorized by location (cortex, white matter, deep nucleus, brainstem, and cerebellum) and region (frontal, parietal, temporal, occipital, insular, hippocampus, corpus callosum, splenium, internal capsule, centrum semiovale, periventricular white matter, globus pallidus, putamen, caudate, thalamus, and cerebellum) [18]. All images were analyzed under cooperation of radiologist and neurologist while blinded to patient's information or development of DNS.

\subsection{Statistical Analysis}

Data are presented as absolute numbers or relative frequencies for categorical variables and medians with interquartile ranges for continuous variables. We compared each variable according to the presence of ABLM and DNS. Variables with a $p$-value of $<0.05$ in univariable analysis were included as candidate variables for multivariable logistic regression modeling. Fisher's exact test and the chi-squared test were used for categorical variables, while the Mann-Whitney test was used for continuous variables. Adjusted odds ratios (ORs) with 95\% confidence intervals (CIs) were also calculated, and two-tailed $p$-values $<0.05$ were considered statistically significant. All statistical analyses were performed using SPSS for Windows version 26 (IBM, Armonk, NY, USA).

\subsection{Modeling}

Significant variables were selected using univariable analysis to evaluate the relationship between the occurrence of DNS and the influencing factors including clinical findings and presence of ABLM classified in terms of pattern, region, and location. The presence of ABLM and brain lesions classified by pattern, region, and location have multicollinearity with each other. So, to avoid multicollinearity problems, we used modelling as follows: Model 1, initial GCS, exposure time, and ABLM; Model 2, initial GCS, exposure time, and ABLM classified by pattern; Model 3, initial GCS, exposure time, and ABLM classified by region; Model 4, initial GCS, exposure time, and ABLM classified by location.

\section{Result}

A total of 283 patients visited our ED because of acute CO poisoning. Of these, 129 were excluded for the following reasons: 10 were under 18 years of age, 27 refused HBO therapy, 51 refused MRI, 11 were discharged against medical advice, 22 were lost to follow-up, 3 had persistent neurological symptoms at discharge, and 5 were transferred from another hospital $24 \mathrm{~h}$ after exposure (Figure 1).

Thus, a total of 154 patients were included in the study. Of the 154 patients, DNS was observed in 30 patients (19.5\%) and the median onset of DNS was 27 days. The DNS group had lower initial GCS score (12.5 vs. $15, p=0.004)$ and longer CO exposure time (360 vs. $150 \mathrm{~min}, p=0.072)$ than the non-DNS group. The DNS group had higher $\mathrm{COHb}(9.3 \mathrm{vs.} 6.0 \%, p=0.139)$, C-reactive protein (CRP) ( 0.23 vs. $0.11 \mathrm{mg} / \mathrm{dL}, p=0.077)$, and lactate $(2.9$ vs. $2.1 \mathrm{mg} / \mathrm{dL}, p=0.690)$ than the non-DNS group. The incidence ratio of ABLM in DNS group was higher than that of non-DNS group ( $60 \%$ vs. $25 \%$, $p=0.001)($ Table 1$)$. 
Table 1. Comparison of baseline characteristics between the non-DNS and DNS groups.

\begin{tabular}{|c|c|c|c|}
\hline & $\begin{array}{l}\text { Non-DNS } \\
(n=124)\end{array}$ & $\begin{array}{c}\text { DNS } \\
(n=30)\end{array}$ & $p$-Value \\
\hline Age, year & $40(29-50.3)$ & $44(35-55)$ & 0.171 \\
\hline Male, $n(\%)$ & $82(66.1)$ & $19(63.3)$ & 0.940 \\
\hline BMI $\left(\mathrm{kg} / \mathrm{m}^{2}\right)$ & $23.3(21.0-25.5)$ & $22.8(21.0-25.4)$ & 0.769 \\
\hline \multicolumn{4}{|l|}{ Comorbidities (\%) } \\
\hline Hypertension & $9(7.3)$ & $3(10.0)$ & 0.902 \\
\hline Diabetes & $2(1.6)$ & $0(0)$ & $>0.999$ \\
\hline \multicolumn{4}{|l|}{ Vital signs } \\
\hline Systolic BP, mmHg & $130(113.5-140)$ & $130(111.3-143.8)$ & 0.744 \\
\hline Diastolic BP, mmHg & $80(70-90)$ & $80(70-90)$ & 0.847 \\
\hline Heart rate, BPM & $92.5(78-105)$ & $92(78.5-100)$ & 0.734 \\
\hline Respiratory rate, $\mathrm{BPM}$ & $20(18.8-20)$ & $20(18-20)$ & 0.215 \\
\hline Body temperature, ${ }^{\circ} \mathrm{C}$ & $36.8(36.5-37.2)$ & $36.9(36.5-37.5)$ & 0.516 \\
\hline $\mathrm{O}_{2}$ saturation, $\%$ & $98(97-98)$ & $97.5(95-98)$ & 0.155 \\
\hline Initial GCS & $15(12-15)$ & $12.5(6.3-15)$ & 0.004 \\
\hline Exposure time, $\min$ & $150(60-315)$ & $360(90-480)$ & 0.072 \\
\hline Time from exposure to MRI, $\mathrm{h}$ & $32(21-48)$ & $43(24-78)$ & 0.175 \\
\hline Current smoking (\%) & $60(50)$ & $15(51.7)$ & $>0.999$ \\
\hline Cause of exposure $(\%)$ & & & 0.124 \\
\hline Accidental & $41(33.1)$ & $5(16.7)$ & \\
\hline Intentional & $83(66.9)$ & $25(83.3)$ & \\
\hline \multicolumn{4}{|l|}{ Symptoms (\%) } \\
\hline Headache & $16(12.9)$ & $2(6.7)$ & 0.524 \\
\hline Loss of consciousness & $31(25)$ & $9(30)$ & 0.743 \\
\hline Chest pain & $2(1.61)$ & $0(0)$ & $>0.999$ \\
\hline Dizziness & $16(12.9)$ & $1(3.3)$ & 0.239 \\
\hline Dyspnea & $5(4.0)$ & $0(0)$ & 0.586 \\
\hline \multicolumn{4}{|l|}{ Laboratory findings } \\
\hline $\mathrm{COHb}, \%$ & $6.0(2.8-11.8)$ & $9.3(4.2-16.7)$ & 0.139 \\
\hline $\mathrm{CRP}, \mathrm{mg} / \mathrm{dL}$ & $0.11(0.1-0.4)$ & $0.23(0.1-0.8)$ & 0.077 \\
\hline Lactate, $\mathrm{mg} / \mathrm{dL}$ & $2.1(1.5-3.9)$ & $2.9(1.8-4.1)$ & 0.690 \\
\hline Troponin I, ng/mL & $0.1(0.1-0.3)$ & $0.1(0.1-0.3)$ & 0.942 \\
\hline Ethanol, mg/dL & $1.5(1.0-7.1)$ & $1.2(0.7-4.2)$ & 0.222 \\
\hline \multicolumn{4}{|l|}{ Outcomes } \\
\hline Onset of DNS, day & - & $27(18-30)$ & - \\
\hline ABLM (\%) & $31(25)$ & $18(60)$ & 0.001 \\
\hline
\end{tabular}

Categorical variables are given as numbers (percentage). Continuous nonparametric variables are given as median (interquartile range). Abbreviations: DNS, delayed neurological sequelae; BMI, body mass index; BP, blood pressure; GCS, Glasgow coma scale; MRI, magnetic resonance imaging; $\mathrm{COHb}$, carboxyhemoglobin; CRP, C-reactive protein; ABLM, acute brain lesion on MRI.

All ABLM patterns were significant $(p<0.05$; Table 2). Significant ABLM regions included the temporal, occipital, and putamen regions $(p<0.05$; Table 2$)$. Among the locations, only white matter lesions were significant; nine patients with DNS (30\%) had a white matter lesion (Table 2, Figure 2). 
Table 2. Lesion distribution according to the development of DNS in patients with ABLM.

\begin{tabular}{|c|c|c|c|}
\hline & \multicolumn{3}{|c|}{ No. (\%) } \\
\hline & Non-DNS & DNS & $p$-Value \\
\hline \multicolumn{4}{|l|}{ Pattern } \\
\hline Globus pallidus lesion & $16(16.2)$ & $11(36.7)$ & 0.031 \\
\hline Diffuse lesion & $13(13.1)$ & $10(33.3)$ & 0.024 \\
\hline Focal lesion & $5(5.1)$ & $7(23.3)$ & 0.007 \\
\hline \multicolumn{4}{|l|}{ Region } \\
\hline Frontal & $5(5.1)$ & $1(3.3)$ & $>0.999$ \\
\hline Parietal & $5(5.1)$ & $3(10)$ & 0.387 \\
\hline Temporal & $2(2.0)$ & $4(13.3)$ & 0.026 \\
\hline Occipital & $2(2.0)$ & $4(13.3)$ & 0.026 \\
\hline Insular & $0(0)$ & $0(0)$ & \\
\hline Hippocampus & $3(3.0)$ & $1(3.3)$ & $>0.99$ \\
\hline Corpus callosum & $3(3.0)$ & $2(6.7)$ & 0.330 \\
\hline Splenium & $2(2.0)$ & $1(3.3)$ & 0.551 \\
\hline Internal capsule & $1(1.0)$ & $0(0)$ & $>0.999$ \\
\hline Centrum semiovale & $3(3.0)$ & $1(3.3)$ & $>0.999$ \\
\hline Periventricular white matter & $1(1.0)$ & $2(6.7)$ & 0.135 \\
\hline Globus pallidus & $16(16.2)$ & $9(30)$ & 0.157 \\
\hline Putamen & $1(1.0)$ & $3(10)$ & 0.039 \\
\hline Caudate & $1(1.0)$ & $0(0)$ & $>0.999$ \\
\hline Thalamus & $2(2.0)$ & $1(3.3)$ & 0.551 \\
\hline Midbrain & $0(0)$ & $0(0)$ & \\
\hline Pons & $0(0)$ & $0(0)$ & \\
\hline Medulla & $0(0)$ & $0(0)$ & \\
\hline Cerebellum & $4(4.0)$ & $1(3.3)$ & $>0.999$ \\
\hline \multicolumn{4}{|l|}{ Location } \\
\hline Cortex & $6(6.1)$ & $4(13.3)$ & 0.240 \\
\hline White matter & $5(5.1)$ & $9(30)$ & $<0.001$ \\
\hline Deep nucleus & $24(24.2)$ & $13(43.3)$ & 0.073 \\
\hline Brainstem & $0(0)$ & $0(0)$ & \\
\hline Cerebellum & $4(4.0)$ & $1(3.3)$ & $>0.999$ \\
\hline
\end{tabular}

When multiple lesions appeared in one person, the numbers were aggregated in duplicate. Abbreviations: DNS, delayed neurological sequelae; ABLM, acute brain lesion on magnetic resonance imaging.

Non-DNS

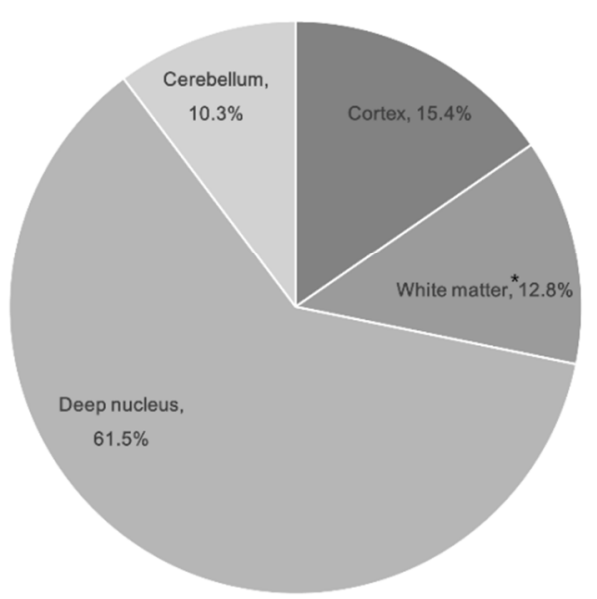

DNS

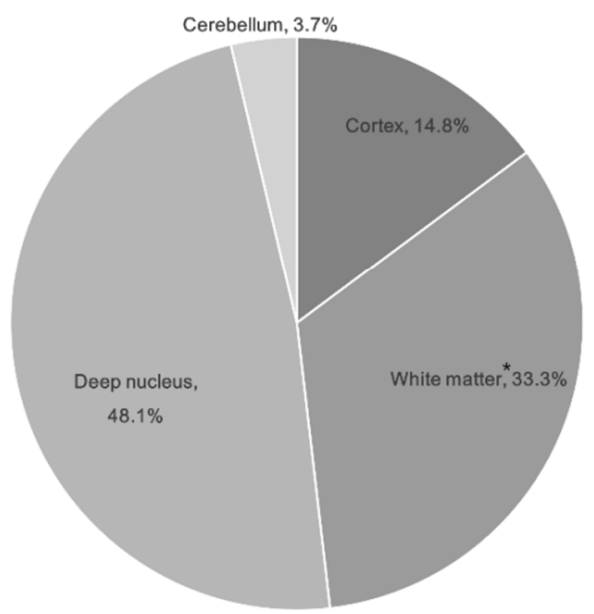

Figure 2. Distribution of brain lesions by location according to the development of delayed neurological sequelae in patients with ABLM. * indicates a significant difference in between the non-DNS and DNS groups ( $p=0.004)$. Abbreviations: ABLM, acute brain lesion on magnetic resonance imaging; DNS, delayed neurological sequelae. 
In the multivariable analysis, four multivariable logistic regression models were used to investigate the significance of categorized brain lesions. The most important variables in each model were as follows: model 1: GCS (OR 0.864; 95\% CI, 0.760-0.983; $p=0.026)$, exposure time (OR 1.002; 95\% CI, $1.001-1.004 ; p=0.010)$, and abnormal MRI (OR 2.242; 95\% CI, 0.864-5.821; $p=0.097)$; model 2: GCS (OR 0.882; 95\% CI, 0.767-1.014; $p=0.078$ ), exposure time (OR 1.002; 95\% CI, 1.001-1.004; $p=0.006)$, and diffuse lesion (OR 2.728; 95\% CI, 0.948-7.850; $p=0.063$ ); model 3: GCS (OR 0.859; 95\% CI, 0.755-0.978; $p=0.022$ ) and exposure time (OR 1.003; 95\% CI, 1.001-1.005; $p=0.001$ ); and model 4: GCS (OR 0.849; 95\% CI, 0.745-0.967; $p=0.014$ ), exposure time (OR 1.003; 95\% CI, 1.001-1.004; $p=0.005$ ), and white matter (OR 6.741; 95\% CI, 1.843-24.660; $p=0.004)$ (Tables 3 and 4).

Table 3. Factors associated with the development of DNS in univariable analysis.

\begin{tabular}{cc}
\hline & $\begin{array}{c}\text { Univariable Analysis } \\
\text { OR (95\% CI) }\end{array}$ \\
\hline COHb, \% & $0.984(0.943-1.027)$ \\
Lactate, mg/dL & $0.992(0.730-1.348)$ \\
CRP, mg/dL & $1.055(0.964-1.155)$ \\
Troponin I, ng/mL & $1.164(0.894-1.516)$ \\
Initial GCS & $0.853(0.771-0.944)$ \\
Exposure time, min & $1.001(1.000-1.002)$ \\
ABLM & $3.804(1.624-8.911)$ \\
Globus pallidus (P) & $3.003(1.203-7.501)$ \\
Diffuse (P) & $3.308(1.270-8.615)$ \\
Focal (P) & $5.722(1.664-19.671)$ \\
Temporal (R) & $7.462(1.294-43.011)$ \\
Occipital (R) & $7.462(1.290-43.010)$ \\
Putamen (R) & $10.889(1.089-108.925)$ \\
White matter (L) & $8.057(2.448-26.516)$ \\
\hline
\end{tabular}

Abbreviations: DNS, delayed neurological sequelae; OR, odds ratio; $\mathrm{CI}$, confidence interval; $\mathrm{COHb}$, carboxyhemoglobin; CRP, C-reactive protein; GCS, Glasgow coma scale; ABLM, acute brain lesion on MRI; $P$, pattern; R, region; L, location.

Table 4. Factors associated with the development of DNS in multivariable analysis.

\begin{tabular}{ccccc}
\hline & \multicolumn{4}{c}{ Multivariable Analysis } \\
\cline { 2 - 5 } & Model 1 & Model 2 & Model 3 & Model 4 \\
& OR (95\% CI) & OR (95\% CI) & OR (95\% CI) & OR (95\% CI) \\
\hline Initial GCS & $0.864(0.760-0.983)$ & $0.882(0.767-1.014)$ & $0.859(0.755-0.978)$ & $0.849(0.745-0.967)$ \\
Exposure time, min & $1.002(1.001-1.004)$ & $1.003(1.001-1.004)$ & $1.003(1.001-1.005)$ & $1.003(1.001-1.004)$ \\
ABLM & $2.242(0.864-5.821)$ & & & \\
Globus pallidus (P) & & $1.160(0.364-3.695)$ & & \\
Diffuse (P) & & $2.728(0.948-7.850)$ & & \\
Focal (P) & & & $1.564(0.527-12.477)$ & \\
Temporal (R) & & $5.643(0.440-72.543)$ & \\
Occipital (R) & & $2.223(0.115-43.033)$ & \\
Putamen (R) & & & $6.741(1.843-24.660)$ \\
White matter (L) & & & & \\
\hline
\end{tabular}

Abbreviations: DNS, delayed neurological sequelae; OR, odds ratio; CI, confidence interval; GCS, Glasgow coma scale; ABLM, acute brain lesion on MRI; P, pattern; R, region; L, location.

\section{Discussion}

There are two types of neurological deficit associated with $\mathrm{CO}$ poisoning: persistent neurologic sequelae that occur immediately after exposure and DNS [19]. DNS manifest with symptoms that occur after a lucid interval of approximately 2-40 days, when the patient has already been discharged without symptoms following initial treatment [20]. Because of this clinical course, it is important to predict DNS early to prevent aggravation. Our study revealed that development of subsequent DNS 
was related to low GCS score, long exposure time, and white matter lesion on MRI; white matter lesion on MRI in particular had a strong association with DNS (OR, 6.741).

\subsection{Incidence of DNS}

DNS occurs in 3-40\% of patients with acute CO poisoning [21,22]. DNS is mediated by inflammatory and immune responses, and there is no established diagnostic method to predict this, resulting in differences in reported DNS incidence [6,23]. The DNS incidence rate in this study was $19.48 \%$, consistent with previous studies.

\subsection{Baseline Characteristics as Predictors for DNS}

Factors such as old age, CO exposure time, cardiac arrest, initial neurological abnormalities, GCS, brain imaging abnormalities, and biochemical indicators (e.g., neuron-specific enolase, CRP, lactic acid) are related to DNS following CO poisoning [4,5]. However, it is controversial whether the above factors can serve as predictors of DNS because the outcomes are inconsistent because of the factors such as the types of patients, the severity of $\mathrm{CO}$ poisoning, and the diversity of outcome measurements.

Moon et al. reported that GCS is a reasonable predictor of DNS [24]. Weaver et al. also noted that if $\mathrm{CO}$ exposure time is long or if there are neurologic symptoms such as loss of consciousness, it is possible to predict the occurrence of DNS [21]. However, it may be impossible or inaccurate to gather an initial GCS or medical history for patients who have attempted suicide through CO poisoning as well as drugs or alcohol, as they may have impaired consciousness [25]. Additionally, patients who attempted suicide are often uncooperative when the medical staff take their history [26]. In our study, 108 (70.13\%) patients admitted with CO poisoning were intentionally exposed. To predict DNS, objective factors other than GCS or history taking were needed. Such objective factors may include laboratory findings and brain imaging.

\subsection{Laboratory Findings as Predictors for DNS}

Biochemical indicators such as troponin I and CRP have been studied as factors to predict the occurrence of DNS following CO poisoning [19,27]. Troponin I is a diagnostic tool for assessing myocardial damage in $\mathrm{CO}$ poisoning but can also be used as a predictor for DNS because increased troponin I indicates reduced blood supply to the brain, which can lead to neurological abnormalities $[27,28]$. However, we found that troponin I was not a predictor for DNS, perhaps because it is a component of the contractile apparatus of myocytes and is too specific to myocardial injury compared with other predictors; thus, it may not accurately reflect pathophysiological differences related to neurological results [28]. Pang et al. [29] reported that CRP can be valuable for predicting DNS, but Jung et al. [23] argued that CRP is not related to the development of DNS, and therefore is inappropriate as a predictor for DNS. In our study, laboratory findings such as troponin I and CRP did not correlate with DNS in univariable logistic regression. According to previous report, symptoms caused by $\mathrm{CO}$ poisoning were proportional to the level of $\mathrm{COHb}$ [30]. However, in our study relatively low level of $\mathrm{COHb}$ was measured compared to previous studies, but several symptoms caused by $\mathrm{CO}$ poisoning appeared and also DNS occurred $[18,19]$. Other studies reported that elevated $\mathrm{COHb}$ play a fundamental diagnostic role in $\mathrm{CO}$ exposure, but it is not correlated with clinical findings and prognosis [6,22]. Because $\mathrm{COHb}$ level is affected by factors such as delay from termination of the $\mathrm{CO}$ exposure to the time blood is drawn for $\mathrm{COHb}$ measurement, as well as intervening administration of oxygen.

\subsection{Brain Imaging as a Predictor for DNS}

Previous studies have explored various imaging tools for predicting DNS following acute CO poisoning but most were found insufficient. Brain lesion on CT scan showed no correlation with the occurrence of DNS in some studies [19,31]. In other studies, electroencephalography and single-photon emission computed tomography (SPECT) were found to be useful in monitoring efficacy of treatment 
but have failed to predict the development of DNS [32,33]. We investigated whether brain MRI, another brain imaging modality, can play a role in predicting DNS following acute $\mathrm{CO}$ poisoning.

Brain lesions caused by $\mathrm{CO}$ poisoning can be seen using MRI in both acute and chronic phases because lesions, which are vulnerable to hypoxic damage, appear in the cerebral cortex, white matter, hippocampus, globus pallidus, and cerebellum [13]. Since MRI can diagnose brain damage from CO poisoning, DNS may be more likely to occur in patients with ABLM. Recent studies have proposed that lesions on MRI, especially on DWIs, can predict DNS [18,34]. However, differences in the locations or patterns of MRI lesions have not revealed any correlations with DNS [13]. We found that ABLM was not correlated with the development of DNS in model 1 of multivariable logistic regression.

The main pathological finding of white matter lesions in DNS is demyelination, and MRI shows high signal intensity in white matter [35]. The lesions in white matter destroy the cortex and neurofibrillary network complex that connects the cortex, resulting in multiple cognitive impairments and balance and gait disorders [11,36]. There have been few reports of lesions in white matter during the acute phase. Moon et al. argued that development of DNS is highly likely if a lesion in white matter is found with MRI conducted within 7 days of CO poisoning [24]. Additionally, Jeon et al. reported that MRIs performed within 1 day did not show differences in lesions between DNS and non-DNS groups [18]. In our study, MRI was performed within 2 days of CO poisoning, and as a result, development of DNS was highly associated with white matter ABLMs.

\subsection{Limitations}

There were some limitations to this study. First, because this was a single-center study, it is difficult to generalize the results. A multicenter study is needed to investigate a larger number of patients through a common measurement method. Second, this study determined only the clinical state without using a formal neurocognitive test when evaluating DNS. This may underestimate cognitive impairments that are difficult to detect or may be biased by the physician. Third, this study revealed that white matter lesions are associated with DNS, but it did not reveal how these lesions were related to patient symptoms (e.g., gait disorder, cognitive disorder). Finally, ABLM manifests differently over time based on the injury. In the case of hypoxic damage, a hyperintense signal can be seen on DWIs up to 7 days after the injury [37]. Further research is needed to investigate changes of brain lesions on MRI over time in the context of CO poisoning.

\section{Conclusions}

We found that lower GCS score, higher exposure time, and the presence of ABLM in white matter are predictors of DNS in patients with acute CO poisoning. Since determining GCS and exposure time accurately may be impossible when there is a loss of consciousness, white matter lesions on MRI may be an important objective factor for early prediction of DNS following CO poisoning.

Author Contributions: Conceptualization, Y.H.L.; formal analysis, S.C.; methodology, J.L., S.-U.L.; investigation, H.B.K.; writing — original draft preparation, S.N.; writing—review and editing, S.H.; supervision, G.W.K., S.H. All authors have read and agreed to the published version of the manuscript.

Funding: This work was supported by the Soonchunhyang University Research Fund grant number 20200021.

Conflicts of Interest: The authors declare no conflict of interest. The funders had no role in the design of the study; in the collection, analyses, or interpretation of data; in the writing of the manuscript, or in the decision to publish the results.

\section{References}

1. Bleecker, M.L. Carbon monoxide intoxication. In Handbook of Clinical Neurology; Elsevier: Amsterdam, The Netherlands, 2015; Volume 131, pp. 191-203.

2. Guzman, J.A. Carbon monoxide poisoning. Crit Care Clin. 2012, 28, 537-548. [CrossRef] [PubMed]

3. Choi, S.-C.; Oh, S. Acute carbon monoxide poisoning and delayed neurological sequelae: A potential neuroprotection bundle therapy. Neural Regen. Res. 2015, 10, 36-38. [CrossRef] [PubMed] 
4. $\quad$ Ku, H.-L.; Yang, K.-C.; Lee, Y.-C.; Lee, M.-B.; Chou, Y.-H. Predictors of carbon monoxide poisoning-induced delayed neuropsychological sequelae. Gen. Hosp. Psychiatry 2010, 32, 310-314. [CrossRef] [PubMed]

5. Hu, H.; Pan, X.; Wan, Y.; Zhang, Q.; Liang, W. Factors affecting the prognosis of patients with delayed encephalopathy after acute carbon monoxide poisoning. Am. J. Emerg. Med. 2011, 29, 261-264. [CrossRef] [PubMed]

6. Thom, S.R.; Taber, R.L.; Mendiguren, I.I.; Clark, J.M.; Hardy, K.R.; Fisher, A.B. Delayed Neuropsychologic Sequelae After Carbon Monoxide Poisoning: Prevention by Treatment With Hyperbaric Oxygen. Ann. Emerg. Med. 1995, 25, 474-480. [CrossRef]

7. Stewart, W.A.; Parent, J.M.-L.; Towner, R.A.; Dobson, H. The use of magnetic resonance imaging in the diagnosis of neurological disease. Can. Vet. J. La Rev. Vet. Can. 1992, 33, 585-590.

8. Teipel, S.J.; Grothe, M.J.; Lista, S.; Toschi, N.; Garaci, F.G.; Hampel, H. Relevance of Magnetic Resonance Imaging for Early Detection and Diagnosis of Alzheimer Disease. Med. Clin. N. Am. 2013, 97, 399-424. [CrossRef]

9. Baird, A.E.; Warach, S. Magnetic Resonance Imaging of Acute Stroke. Br. J. Pharmacol. 1998, 18, 583-609. [CrossRef]

10. Van Everdingen, K.J.; Van Der Grond, J.; Kappelle, L.; Ramos, L.; Mali, W. Diffusion-weighted magnetic resonance imaging in acute stroke. Stroke 1998, 29, 1783-1790. [CrossRef]

11. Parkinson, R.B.; Hopkins, R.O.; Cleavinger, H.B.; Weaver, L.K.; Victoroff, J.; Foley, J.F.; Bigler, E.D. White matter hyperintensities and neuropsychological outcome following carbon monoxide poisoning. Neurology 2002, 58, 1525-1532. [CrossRef]

12. Weaver, L.K.; Orrison, W.W.; Deru, K.; McIntosh, J. Brain imaging abnormalities in carbon monoxide-poisoned patients with ongoing symptoms at least 6 months after poisoning. Undersea Hyperb Med. Soc. 2015, 100, 39.

13. O'Donnell, P.; Buxton, P.; Pitkin, A.; Jarvis, L. The Magnetic Resonance Imaging Appearances of the Brain in Acute Carbon Monoxide Poisoning. Clin. Radiol. 2000, 55, 273-280. [CrossRef] [PubMed]

14. Lo, C.-P.; Chen, S.-Y.; Lee, K.-W.; Chen, W.-L.; Chen, C.-Y.; Hsueh, C.-J.; Huang, G.-S. Brain Injury after Acute Carbon Monoxide Poisoning: Early and Late Complications. Am. J. Roentgenol. 2007, 189, W205-W211. [CrossRef]

15. Lim, P.J.; Shikhare, S.N.; Peh, W.C.G. Clinics in diagnostic imaging (154). Carbon monoxide (CO) poisoning. Singapore Med. J. 2014, 55, 405-409. [CrossRef]

16. Weaver, L.; Hopkins, R.O.; Chan, K.J.; Churchill, S.; Elliott, C.G.; Clemmer, T.P.; Orme, J.F.; Thomas, F.O.; Morris, A.H. Hyperbaric Oxygen for Acute Carbon Monoxide Poisoning. N. Engl. J. Med. 2002, 347, 1057-1067. [CrossRef]

17. Ide, T.; Kamijo, Y.; Ide, A.; Yoshimura, K.; Nishikawa, T.; Soma, K.; Mochizuki, H. Elevated S100B level in cerebrospinal fluid could predict poor outcome of carbon monoxide poisoning. Am. J. Emerg. Med. 2012, 30, 222-225. [CrossRef]

18. Jeon, S.-B.; Sohn, C.H.; Seo, N.-W.; Oh, B.J.; Lim, K.S.; Kang, D.-W.; Kim, W.Y. Acute Brain Lesions on Magnetic Resonance Imaging and Delayed Neurological Sequelae in Carbon Monoxide Poisoning. JAMA Neurol. 2018, 75, 436-443. [CrossRef]

19. Kudo, K.; Otsuka, K.; Yagi, J.; Sanjo, K.; Koizumi, N.; Koeda, A.; Umetsu, M.Y.; Yoshioka, Y.; Mizugai, A.; Mita, T.; et al. Predictors for delayed encephalopathy following acute carbon monoxide poisoning. BMC Emerg. Med. 2014, 14, 3. [CrossRef] [PubMed]

20. Sönmez, B.M.; İşcanlı, M.D.; Parlak, I.S.; Doğan, Y.; Ulubay, H.G.; Temel, E. Delayed neurologic sequelae of carbon monoxide intoxication. Turk. J. Emerg. Med. 2018, 18, 167-169. [CrossRef]

21. Weaver, L.K.; Valentine, K.J.; Hopkins, R.O. Carbon Monoxide Poisoning. Am. J. Respir. Crit. Care Med. 2007, 176, 491-497. [CrossRef]

22. Pepe, G.; Castelli, M.; Nazerian, P.; Vanni, S.; Del Panta, M.; Gambassi, F.; Botti, P.; Missanelli, A.; Grifoni, S. Delayed neuropsychological sequelae after carbon monoxide poisoning: Predictive risk factors in the Emergency Department. A retrospective study. Scand. J. Trauma Resusc. Emerg. Med. 2011, 19, 16. [CrossRef] [PubMed]

23. Jung, J.W.; Lee, J.H. Serum lactate as a predictor of neurologic outcome in ED patients with acute carbon monoxide poisoning. Am. J. Emerg. Med. 2019, 37, 823-827. [CrossRef] [PubMed]

24. Moon, G.J.; Kim, G.T. Acute carbon monoxide poisoning: Prognostic factors for delayed neuropsychiatric sequelae. J. Korean Soc. Emerg. Med. 2014, 25, 268-276. 
25. Hampson, N.B.; Bodwin, D. Toxic CO-ingestions in Intentional Carbon Monoxide Poisoning. J. Emerg. Med. 2013, 44, 625-630. [CrossRef]

26. Lee, H.-L.; Lin, H.-J.; Yeh, S.-Y.; Chi, C.-H.; Guo, H.-R. Etiology and outcome of patients presenting for poisoning to the emergency department in Taiwan: A prospective study. Hum. Exp. Toxicol. 2008, 27, 373-379. [CrossRef]

27. Kao, H.-K.; Lien, T.-C.; Kou, Y.R.; Wang, J.-H. Assessment of myocardial injury in the emergency department independently predicts the short-term poor outcome in patients with severe carbon monoxide poisoning receiving mechanical ventilation and hyperbaric oxygen therapy. Pulm. Pharmacol. Ther. 2009, 22, 473-477. [CrossRef]

28. Moon, J.M.; Chun, B.J.; Shin, M.-H.; Lee, S. Serum N-terminal proBNP, not troponin I, at presentation predicts long-term neurologic outcome in acute charcoal-burning carbon monoxide intoxication. Clin. Toxicol. 2017, 56, 412-420. [CrossRef]

29. Pang, L.-X.; Wang, H.-L.; Wang, Z.-H.; Wu, Y.; Dong, N.; Xu, D.-H.; Wang, D.-W.; Xu, H.; Zhang, N. Plasma copeptin as a predictor of intoxication severity and delayed neurological sequelae in acute carbon monoxide poisoning. Peptides 2014, 59, 89-93. [CrossRef] [PubMed]

30. Weaver, L.K. Carbon monoxide poisoning. N. Engl. J. Med. 2009, 360, 1217-1225. [CrossRef] [PubMed]

31. Choi, I.S.; Kim, S.K.; Choi, Y.C.; Lee, S.S.; Lee, M.S. Evaluation of outcome after acute carbon monoxide poisoning by brain CT. J. Korean Med. Sci. 1993, 8, 78-83. [CrossRef]

32. Watanabe, S.; Asai, S.; Sakurai, I.; Suzuki, Y.; Yamamoto, I.; Inokuchi, S.; Murase, H.; Miyachi, H. Analysis of basic activity of electroencephalogram in patients with carbon monoxide intoxication for monitoring efficacy of treatment. Rinsho Byori Jpn. J. Clin. Pathol. 2006, 54, 1199-1203.

33. Chen, S.-Y.; Lin, C.-C.; Lin, Y.-T.; Lo, C.-P.; Wang, C.-H.; Fan, Y.-M. Reversible Changes of Brain Perfusion SPECT for Carbon Monoxide Poisoning-Induced Severe Akinetic Mutism. Clin. Nucl. Med. 2016, 41, e221-e227. [CrossRef] [PubMed]

34. Kim, Y.-S.; Cha, Y.S.; Kim, M.S.; Kim, H.; Lee, Y.S.; Youk, H.I.; Kim, H.; Kim, O.H.; Cha, K.-C.; Lee, K.H.; et al. The usefulness of diffusion-weighted magnetic resonance imaging performed in the acute phase as an early predictor of delayed neuropsychiatric sequelae in acute carbon monoxide poisoning. Hum. Exp. Toxicol. 2017, 37, 587-595. [CrossRef]

35. Lapresle, J.; Fardeau, M. The Central Nervous System and Carbon Monoxide Poisoning II. Anatomical Study of Brain Lesions following Intoxication with Carbon Monoxide (22 cases). In Progress in Brain Research; Elsevier: Amsterdam, The Netherlands, 1967; Volume 24, pp. 31-74. [CrossRef]

36. Guo, A.C.; MacFall, J.R.; Provenzale, J.M. Multiple Sclerosis: Diffusion Tensor MR Imaging for Evaluation of Normal-appearing White Matter. Radiology 2002, 222, 729-736. [CrossRef]

37. Allen, L.M.; Hasso, A.N.; Handwerker, J.; Farid, H. Sequence-specific MR Imaging Findings That Are Useful in Dating Ischemic Stroke. Radiographics 2012, 32, 1285-1297. [CrossRef] 